\section{Transgastric endoscopic ultrasound (EUS)-guided gallbladder drainage for acute cholecystitis}

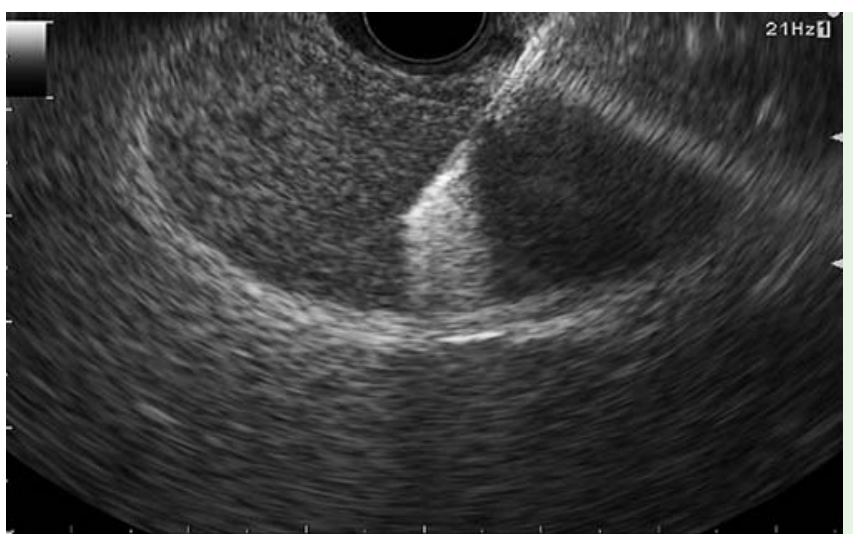

Fig. 1 Gallbladder punctured under endoscopic ultrasound guidance before placement of the guide wire.

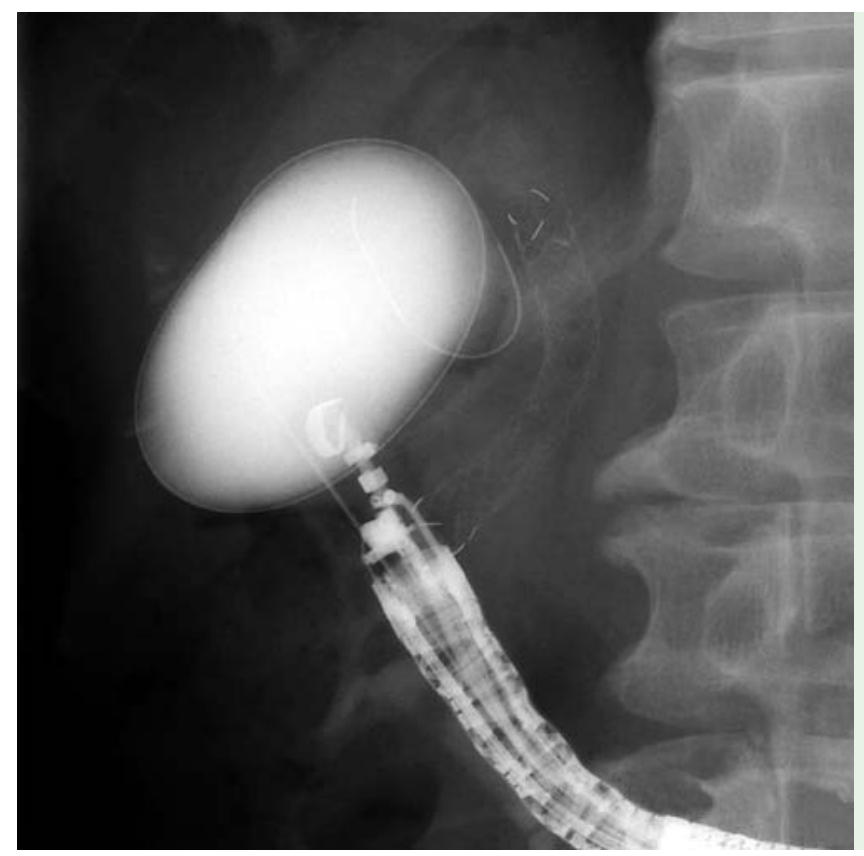

Fig. 2 Fluoroscopic image showing the coiled guide wire in the gallbladder.

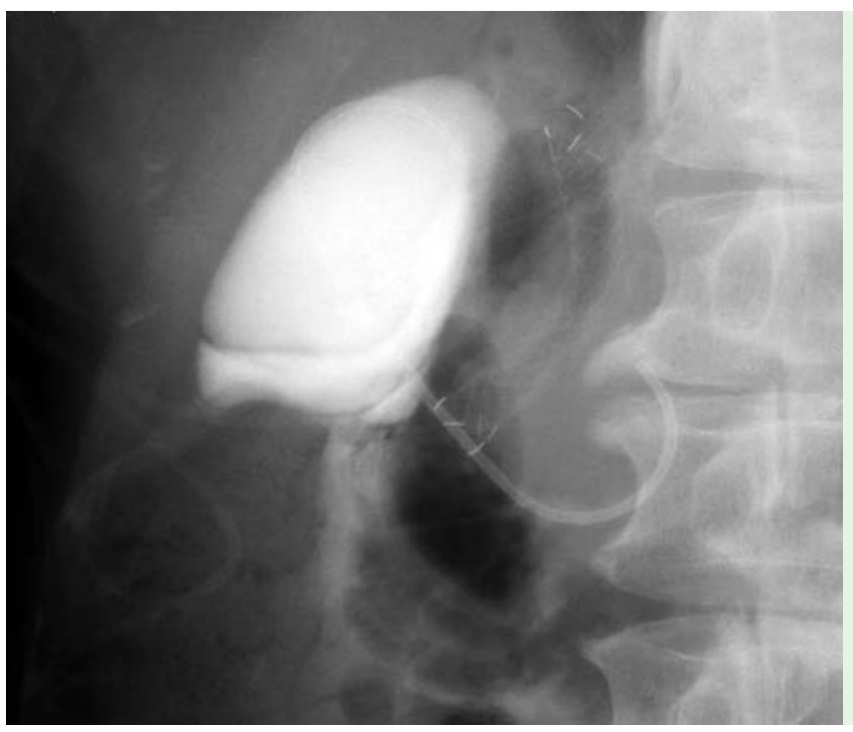

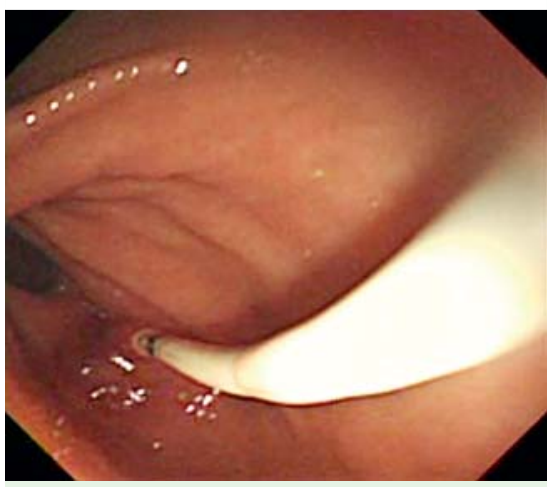

Fig. 4 Endoscopic view of the stent inserted into the gallbladder from the antrum of the stomach.

Acute cholecystitis occurs in $4 \%-7 \%$ of patients with a covered metallic stent (CMS) placed in the bile duct $[1,2]$. Percutaneous transhepatic gallbladder drainage, which involves an external drainage tube, decreases the ability of the patient to carry out their normal daily activities. Recently, endoscopic ultrasound (EUS)guided drainage has been employed successfully for hepatogastrostomy, bilioduodenostomy, and pancreatogastrostomy [3-5]. We report here a patient who underwent EUS-guided gallbladder drainage for acute cholecystitis caused by CMS placement.

A 71-year-old man with unresectable pancreatic cancer underwent deployment of a CMS for obstructive jaundice. On the eighth post-procedure day, he complained of abdominal pain and developed fever, associated with an increase in white blood cell counts and raised serum level of C-reactive protein. Computed tomography revealed an enlarged gallbladder, suggesting acute cholecystitis and requiring continuous drainage of the gallbladder. Therefore, after obtaining informed consent, we carried out EUS-guided gallbladder drainage. An echoendoscope (GF-UCT240-AL5; Olympus, Tokyo, Japan) was introduced into the stomach, and a 19-gauge needle (Echo-Tip; Wilson-Cook, Winston-Salem, North Carolina, USA) was used to puncture the gallbladder ( Fig. 1) and create a gastro-gallbladder fistula. The infected bile was immediately aspirated via the needle and the gallbladder was irrigated with a contrast medium containing an antibiotic. A 0.035-inch guide wire (Revowave, Olympus, Tokyo, Japan) was passed through the needle under fluoroscopic guidance until it reached the gallbladder; the guide wire was coiled within the gallbladder ( $\bullet$ Fig. 2). Three biliary dilation catheters (6 Fr, 7 Fr, and $9 \mathrm{Fr}$; Soehendra 
Biliary Dilation Catheters, Wilson-Cook, Winston-Salem, North Carolina, USA) were serially advanced over the guide wire to dilate the diameter of the tract. A pigtail stent (diameter $7 \mathrm{Fr}$; length $4 \mathrm{~cm}$ ) was placed over the guide wire to bridge the gallbladder and the antrum of the stomach ( Fig. 3,4 ). There were no procedure-related complications. The patient's fever and abdominal pain resolved rapidly and laboratory data showed improvement 5 days later. Although the stent was kept in place for 6 months without any additional intervention, such as removal or exchange of the stent, there were no recurrent symptoms.

Endoscopy_UCTN_Code_TTT_1AS_2AD

\section{K. Kamata, M. Kitano, T. Komaki, H. Sakamoto, M. Kudo}

Division of Gastroenterology and Hepatology, Department of Internal Medicine, Kinki University School of Medicine,

Oasaka-sayama, Japan

\section{References}

1 Maria S, Plinio R, Renon $U$ et al. Malignant biliary obstruction: Treatment with ePTFEFEP-covered endoprostheses-initial technical and clinical experiences in a multicenter trial. Radiology 2002; 225: 35-42

2 Isayama H, Komatsu Y, Tsujino T et al. A prospective randomised study of covered versus uncovered diamond stents for the management of distal malignant biliary obstruction. Gut 2004; 53: 729-734

3 Burmester E, Niehaus J, Leineweber T et al. EUS-cholangio-drainage of the bile duct: report of 4 cases. Gastrointest Endosc 2003; 57: $246-251$
4 Giovannini M, Moutardier V, Pesenti C et al. Endoscopic ultrasound-guided bilioduodenal anastomosis: a new technique for biliary drainage. Endoscopy 2001; 33: 898-900

5 Francois E, Kahaleh M, Giovannini $M$ et al. EUS-guided pancreaticogastrostomy. Gastrointest Endosc 2002; 56: 128 - 133

\section{Bibliography}

DOI $10.1055 / \mathrm{s}-0029-1215225$

Endoscopy 2009; 41: E315-E316

(c) Georg Thieme Verlag KG Stuttgart · New York . ISSN 0013-726X

\section{Corresponding author}

\section{Kitano, MD, PhD}

Division of Gastroenterology and Hepatology

Kinki University School of Medicine

Ohno-higashi 377-2

Osaka-sayama 589-8511

Japan

Fax: +81-72-3660221

m-kitano@med.kindai.ac.jp 